\title{
Temporal Peaks Restoration: A New Innovation
}

\author{
Muhammad Ahmad \\ Cosmetic Plastic and Hair Restorative \\ Surgeon, La Chirurgie, Islamabad \\ Cosmetic Surgery Centre, Islamabad, \\ Pakistan
}

No potential conflict of interest relevant to this article was reported.
There are several methods for restoring the temporal peaks. The most important point is the variable density and angulation of the hair, which ultimately give a soft and natural look. The current technique produces zones of variable density and helps to restore naturalness.

Keywords Alopecia, Hair, Hair follicle

\section{INTRODUCTION}

Restoration of the normal temporal area is an essential part of modern hair restoration, but doing so is technically very difficult. That is why, in the past, hair restoration surgeons were reluctant to restore this area [1]. Various systems for classification of temporal point recession are available [2,3]. The male temporal area normally consists of a concave lower border whose superior margin forms the temporal point [4]. A different degree of recession may be noted on the right and left sides, which further stresses the need to restore the temporal peaks to balance the face [5]. The recreation of the anterior hairline without addressing the area of the temporal peaks produces the unnatural effect of wearing a hairpiece [4]. Although the temporal hair is not dense, the angles of the exiting hair are very acute $\left(10^{\circ}\right.$ or less), which creates problems for the restoration surgeon. There is also a change in the hair direction in the upper and lower parts of the temporal peaks.

The author hereby presents his technique for addressing the temporal areas.

\section{ANATOMICAL FACTORS}

The author has noted that the direction of hair in the temporal area is different on the right and left side. This may be related to the lo-

Received: Mar 12, 2016 Revised: May 26, 2016 Accepted: Jun 8, 2016 Correspondence: Muhammad Ahmad Plastic \& Hair Restorative Surgeon, Hair Transplant Institute, H.1-B, Street 16, Main Kohistan Road, Sector F-8/3, Islamabad, Pakistan. E-mail: platicsurgeonpk@yahoo.com

Copyright @ 2016 The Korean Society for Aesthetic Plastic Surgery.

This is an Open Access article distributed under the terms of the Creative Commons Attribution Non-Commercial License (http://creativecommons.org/licenses/by-nc/4.0/) which permits unrestricted non-commercial use, distribution, and reproduction in any medium, provided the original work is properly cited. www.e-aaps.org cation of the scalp whorl, but no study to date is available to verify this clinical observation.

\section{PROPOSED ZONES RECONSTRUCTION}

The author has divided the temporal peaks area into three zones (I, II and III). Zone I includes the first $1 \mathrm{~cm}$ of the area to be reconstructed. It has an upper limb and a lower limb. Zone II is 1 to $2 \mathrm{~cm}$ in width and follows zone I (Fig. 1). Zone III is triangular in shape and lies next to zone II.

Only single-hair containing follicular units (FUs) with fine hairs should be used in zone I. The density may vary from 10 to 15 per $\mathrm{cm}^{2}$. In zone II, single-hair containing FUs should be used, but the density should be slightly higher than in zone I (i.e., 15 to 20 per $\mathrm{cm}^{2}$ ). In zone III, 1- or 2-hair FUs should be used to match the density of the pre-existing temporal hair $\left(25\right.$ to $\left.30 \mathrm{per} \mathrm{cm}^{2}\right)$. Only singlehair FUs should be used in zone I and II so that in the worst case scenario, if these hairs fall out due to aging, the 2-hair FUs would be hidden, as even one of the 2-hair FUs may create an unnatural look (Fig. 1).

\section{DISCUSSION}

Hair restoration surgeons have various techniques they have personally developed to recreate the temporal peaks. For example, the use of hair from the nape of the neck or the use of a laser, which can alter the characteristics of the hair, both give the area a softer look [6]. The most important point in temporal hair recreation is the angle of the hair shaft exit. Every effort must be made to keep the angles as acute as possible ( $10^{\circ}$ or less). The author uses superinflation with saline in the superficial skin layers. The thumb is used to press the skin down like a fulcrum of lever. This maneuver lifts the distal part of the skin where the blade enters the skin to 


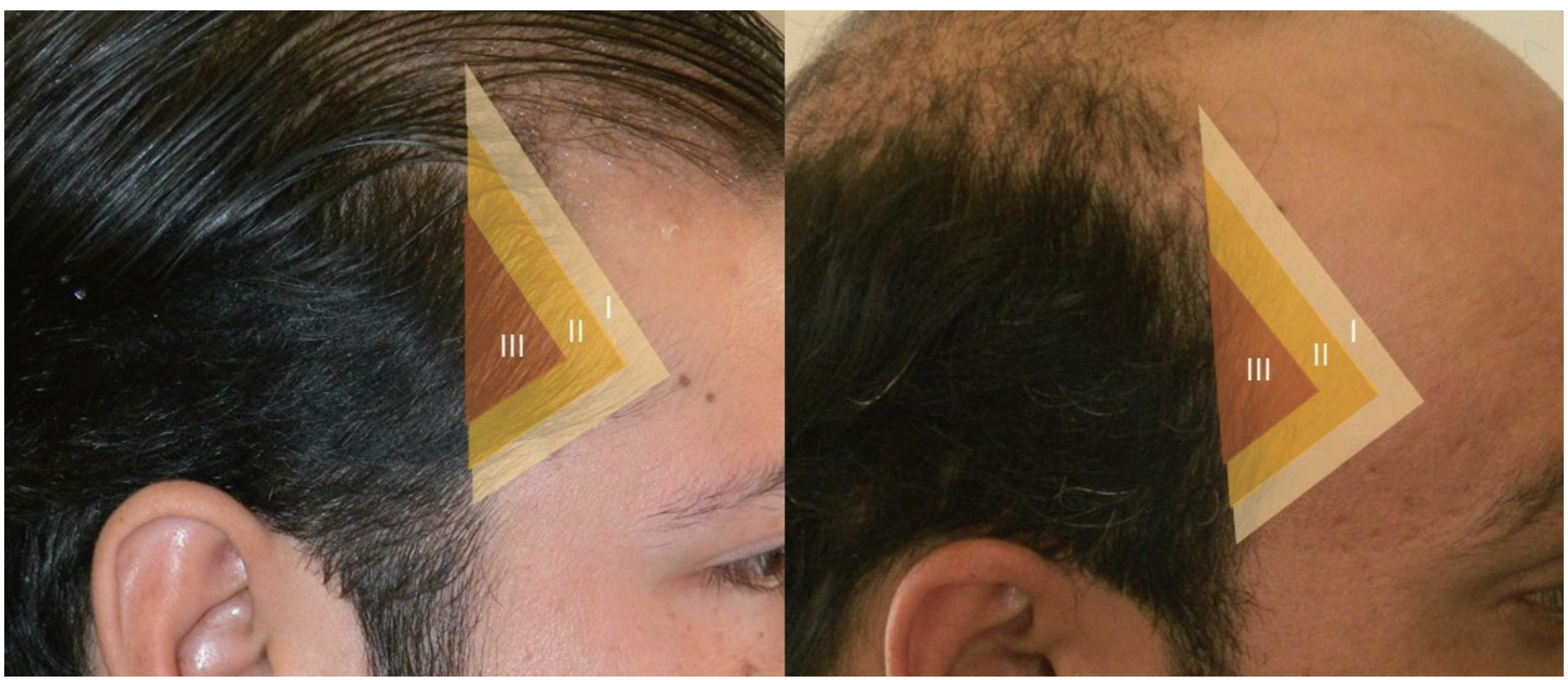

Fig. 1. Zones of temporal peaks.

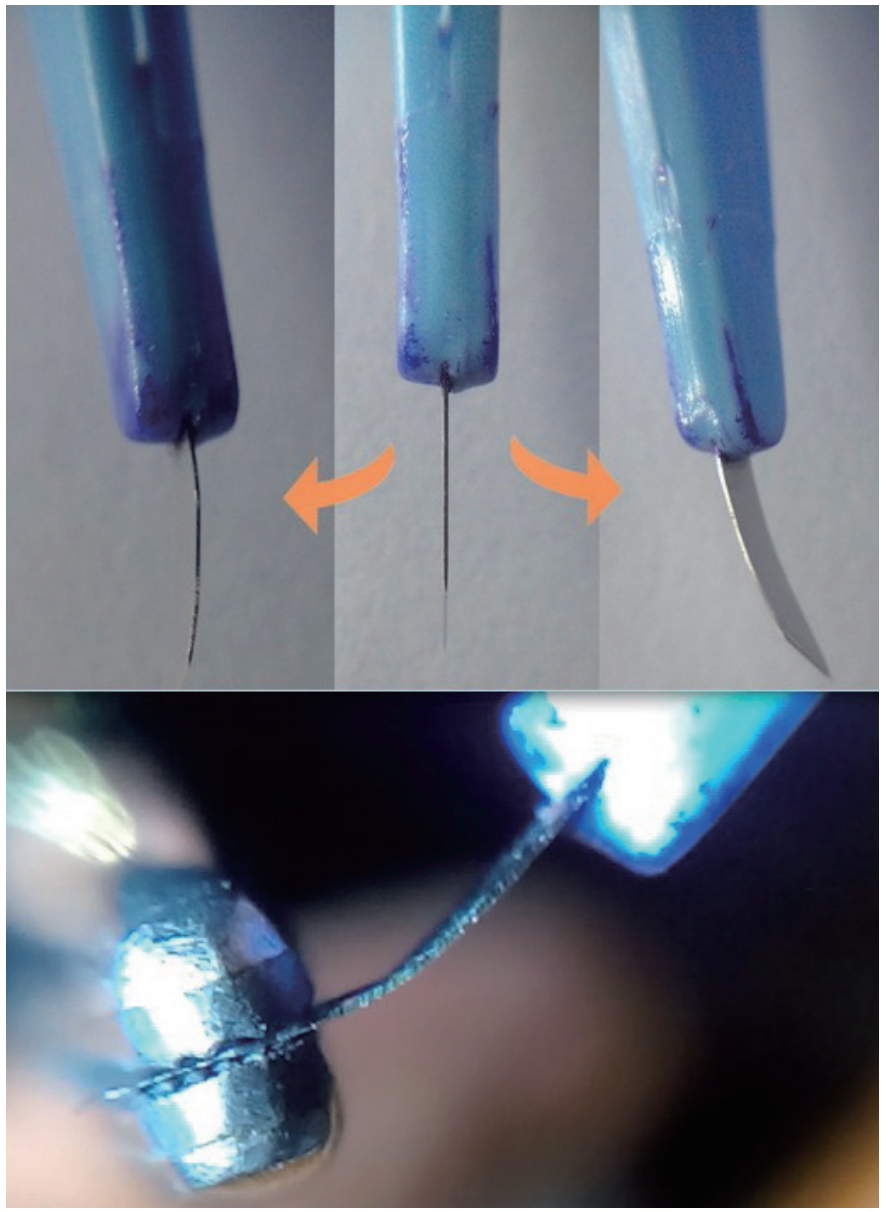

Fig. 2. Maneuvering of the blade. make the slits. In addition, the blade is also turned slightly, in such a way that the concave side of the blade faces away from the nose of the patient while making slits (Fig. 2). This is achieved by holding the blade in the needle holder and gently twisting the blade, resulting in a slight curve in the blade. Care is taken not to make the curve very acute, as it may break the blade during twisting or during the process of slit creation. This maneuver provides a 3D rotation to the exiting hair shaft. Moreover the curvature of the shaft should always remain towards the skin [7]. In FU extraction, because the hairs are trimmed to 1 to $2 \mathrm{~mm}$, it becomes very difficult to determine the angle of curvature of the hair shaft. In these circumstances, the current technique will result in a quality outcome.

\section{CONCLUSION}

This technique was found to be very practical and useful in recreation of the temporal peaks.

\section{PATIENT CONSENT}

Patients provided written consent for the use of their images.

\section{REFERENCES}

1. Unger WP. Hair transplantation. 3rd ed. New York, NY: Marcel Dekker, Inc.; 1995.

2. Brandy DA. A method for evaluating and treating the temporal peak region in patients with male pattern baldness. Dermatol Surg 2002;28: 394-400.

3. Mayer ML, Perez-Meza D. Temporal points classification and surgical 
techniques. ESHRS J 2002;3:6-7.

4. Parsley WM. Natural hair patterns. Facial Plast Surg Clin North Am 2004; $12: 167-80$.

5. Wong J. When to restore the temple area. In: Pathomvanich D, Imagawa K, editors. Hair Restoration Surgery in Asians. Tokyo, JP: Springer; 2010. p.47-8.
6. Mohmand MH. Hair curvature: an important factor for a natural looking hairline. ESHRS J 2005;5:6-7.

7. Umar S. Use of nape and peri-auricular hair by follicular unit extraction to create soft hairlines and temples: my experience with 128 patients. Aesthet Surg J 2015;35:903-9. 